\title{
O grupo multiplicativo de uma álgebra com divisão
}

Daniele Cristina Gualti Scalabrini

\author{
DISSERTAÇÃO APRESENTADA \\ $\mathrm{AO}$ \\ INSTITUTO DE MATEMÁTICA E ESTATÍSTICA \\ DA \\ UNIVERSIDADE DE SÃO PAULO, \\ PARA \\ OBTENÇÃO DO TÍTULO DE MESTRE \\ EM \\ CIÊNCIAS
}

Área de concentração: Matemática
Orientadora: Profa. Dra. Leila Maria Vasconcellos Figueiredo

Durante o desenvolvimento deste trabalho, a autora recebeu apoio financeiro da Instituição mantenedora da Faculdade de Ciências Gerencias de Dracena,

o Centro de Ensino Superior de Dracena - CESD

São Paulo

2003 


\title{
O grupo multiplicativo \\ de uma álgebra \\ com divisão
}

\author{
Este exemplar corresponde à redação \\ final da dissertação devidamente corrigida \\ e defendida por Daniele Cristina Gualti Scalabrini \\ e aprovada pela comissão julgadora.
}

São Paulo, 12 de maio de 2003.

Banca examinadora:

- Profa. Dra. Leila Maria Vasconcellos Figueiredo (Orientadora) (IME-USP)

- Prof. Dr. Jairo Zacarias Gonçalves (IME-USP)

- Prof. Dr. Guilherme Augusto de La Rocque Leal (UFRJ) 
Aos meus pais, meu marido e filho, pelo apoio e confiança durante todos estes anos. 


\section{Resumo}

Como uma generalização do clássico Teorema de Wedderburn, mostramos que o grupo multiplicativo de uma álgebra com divisão não comutativa de dimensão finita sobre seu centro, não pode ser finitamente gerado.

Também estudamos algumas propriedade de um anel com divisão $D$, em que os elementos do grupo derivado $D^{\prime}$ (subgrupo dos comutadores ) de $D^{*}=D-\{0\}$, são algébricos sobre o centro $F$ de $D$ e concluímos que, se cada elemento de $D^{\prime}$ é algébrico sobre $F$, então, $D$ é algébrico sobre $F$. 


\section{Abstract}

As a generalization of Wedderburn's classic theorem, we show that the multiplicative group of a noncommutative finite dimensional division algebra over its center, cannot be finitely generated.

We also studied some properties of a division ring $D$, in which the elements of the derived group $D^{\prime}$ of $D^{*}=D-\{0\}$, are algebraic over the center $F$ of $D$ and we conclude that, if each element of $D^{\prime}$ is algebraic over $F$, then, $D$ is algebraic over $F$. 


\section{Agradecimentos}

A Deus, por propiciar o ensejo de crescer e realizar mais esta conquista.

Aos meus pais, Luiz Natal e Gracinda, anjos iluminados, pela coragem e dedicação despendidas na criação de todos os filhos, pelo especial apoio dado a mim, pela inestimável ajuda prestada, prinicipalmente durante todos esses anos de estudos intensos e ingentes.

Ao Professor José Gonzaga da Silva Neto, eminente Educador, por ter acreditado em mim, pelo apoio irrestrito e pelo seu instinto paterno que o leva a proporcionar relevantes oportunidades aos seus colaboradores.

À minha Orientadora, Professora Doutora Leila Maria Vasconcellos Figueiredo, por seu acolhimento fraternal, por suas contribuições sábias, pela dedicação e sincera amizade.

Aos colegas do IME, que muito contribuiram com sugestões, ajudando-me a dirimir as constantes dúvidas.

Aos meus Coordenadores, Mestres Célio Rejani e Marcelo Plens e aos Professores, Dr. Alfredo Peixoto Martins e Dr. Élio Takeshi Tachizawa pelas contribuições.

Ao Professor Norberto Barboza Crispim, meu ilustre Professor de Português, pelas correções necessárias e pela participação enriquecedora.

Às Instituições Mantenedoras, Centro de Ensino Superior de Dracena - CESD - e Centro de Ensino Superior de Tupi Paulista - CESTUPI - pelo suporte financeiro e moral que me ofereceram durante todo o tempo em que desenvolvi este trabalho.

À minha sogra, Elena Alves Scalabrini, pela ajuda, principalmente nas horas de grandes correrias.

Aos meus irmãos, familiares e amigos que sempre me incentivaram e que, agora, partilham comigo desta conquista.

Ao meu esposo, Marcos, pelo constante estímulo, apoio, dedicação, paciência e compreensão, e que também se privou de muitas coisas a fim de me respaldar nesta conquista.

Ao meu filho, João Pedro, meiga criança, que tão cedo foi privado de minha presença para que eu pudesse adentrar de corpo, alma, coração e afeto, neste trabalho.

A todos, inclusive àqueles que se perderam na memória dos tempos, o meu eterno "Muito Obrigado!" 


\section{Sumário}

$\begin{array}{ll}\text { Introdução } & 8\end{array}$

1 Preliminares 10

2 O Grupo Multiplicativo de uma Álgebra com Divisão 17

3 O Grupo Derivado de um Anel com Divisão 21

$\begin{array}{lr}\text { Bibliografia } & 29\end{array}$ 


\section{Introdução}

A pergunta mais geral de que grupos podem ocorrer como grupos multiplicativos de uma álgebra com divisão não comutativa é, ainda, um problema não resolvido, mas muito progresso tem sido feito nesta linha.

Tudo começou em 1905 com o Teorema de Wedderburn, que demonstrou que todo anel com divisão finito é comutativo. Durante os anos de 1940, 1950, muita pesquisa foi feita no sentido de descobrir que tipo de condições que, quando impostas ao grupo multiplicativo de um anel com divisão, implicam em sua comutatividade, como por exemplo, o resultado encontrado por N. Jacobson, generalizando o Teorema de Wedderburn, o qual diz que um anel $\mathrm{R}$ tal que $x^{n}=x$, para algum inteiro $n=n(x)>1$ e para todo $x$ em $\mathrm{R}$, é comutativo.

No entanto, são poucos os anéis comutativos que verificam as hipóteses do Teorema de Jacobson e, por isso, tentou-se estender este resultado em outras direções de modo que as condições impostas sobre o anel, sejam verificadas por todos os anéis comutativos e ainda, que elas forcem a comutatividade dos anéis em questão. Assim, desde o início da década de 50, os Teoremas de Comutatividade começaram a ser estudados por I. Herstein, C. Faith, I. Kaplansky, Hua e outros.

No estudo da comutatividade surgiu, naturalmente, a idéia de retirar informações do grupo multiplicativo dos anéis. Quanto maior fosse o centro do anel em relação a seu grupo multiplicativo, mais perto estaríamos de obter um grupo comutativo e, portanto, um corpo.

Para mostrarmos isso, vamos fazer uma sucinta exposição sobre os principais teoremas nessa área que foram algumas das conseqüências do Teorema de Wedderburn, como os teoremas de comutatividade em anéis com divisão com condições impostas a comutadores aditivos e multiplicativos, incluindo o Lema de Herstein e o Teorema de Brauer-Cartan-Hua.

No Capítulo 1, apresentamos alguns teoremas de comutatividade e definições que serão utilizados na demonstração dos teoremas que virão a seguir.

No capítulo 2, temos um dos últimos resultados nessa linha, que é o resultado principal dessa dissertação: o teorema que diz que, se $D$ é uma álgebra com divisão de dimensão finita sobre seu centro, e se $D^{*}=D-\{0\}$, o grupo multiplicativo de $D$, é 
finitamente gerado, então $D$ é comutativa.

No capítulo 3, nos preocupamos com o grupo derivado $D^{\prime}$ de $D^{*}$ onde obtivemos que, se em um anel com divisão $D$ o grupo $D^{\prime}$ é algébrico sobre um subcorpo finito de $D$, então $D$ é comutativo. Esse teorema é uma generalização do Teorema de Jacobson que diz que todo anel com divisão algébrico sobre um corpo finito é comutativo, além de outros resultados interessantes nessa área. 


\section{Capítulo 1}

\section{Preliminares}

Neste capítulo apresentaremos alguns resultados básicos da Teoria dos Anéis que surgiram no estudo de condições sobre o anel que implicassem em sua comutatividade. Para isso, temos também que introduzir algumas definições. A maioria das demonstrações dos resultados aqui enunciados encontra-se em [ 7]. Denotaremos sempre por $R$ um anel associativo, não necessariamente com unidade; $D$ será um anel com divisão com centro $F$, cujos grupos multiplicativos serão denotados por $D^{*}$ e $F^{*}$, respectivamente.

Tentando responder à pergunta de que grupos podem ocorrer como grupos multiplicativos de uma álgebra com divisão não comutativa, obteve-se muitos resultados importantes, como por exemplo, o resultado provado por Hua, que mostrou que o grupo multiplicativo de uma álgebra com divisão não comutativa, não pode ser solúvel.

O Teorema de Wedderburn, provado em 1905, foi o primeiro resultado na linha de comutatividade, demonstrando que todo anel com divisão finito é um corpo. Durante os anos de 1940, 1950, muita pesquisa foi feita no sentido de descobrir que tipo de condições que, quando impostas ao grupo multiplicativo de um anel com divisão, implicam em sua comutatividade.

Para os algebristas esse teorema funcionou como ponto de partida para uma extensa área de pesquisa relacionada com comutatividade de anéis. Tentaram, por um lado, substituir a finitude por outras condições que implicassem comutatividade e, por outro, substituir anel com divisão por anéis mais gerais.

Como exemplos de generalizações do teorema de Wedderburn temos as seguintes proposicões e teoremas interessantes:

Proposição 1.1 Qualquer subanel finito $R$, de um anel com divisão $D$, é um corpo.

Proposição 1.2 Seja $D$ um anel com divisão de característica $p>0$, e $G$ subgrupo finito de $D^{*}$. Então, $G$ é cíclico.

Proposição 1.3 Seja D um anel com divisão. Se um elemento y de D comuta com todos comutadores aditivos em $D$, então, y está no centro do anel. 
Proposição 1.4 Seja $D$ um anel com divisão. Se todos os comutadores aditivos estão no centro de $D$, então, $D$ é um corpo.

Definição 1.5 Sejam $R$ um anel e a um elemento de $R$. Seja $d_{a}: R \rightarrow R$ a função definida por $d_{a}(x)=a x-x a$, para $x \in R$. Então:

$$
d_{a}(x+y)=d_{a}(x)+d_{a}(y)
$$

$e$

$$
d_{a}(x y)=x d_{a}(y)+d_{a}(x) y
$$

para todos $x, y \in R$. Chamamos $d_{a}$ de derivação interna de $R$ associada ao elemento a de $R$. Um subgrupo aditivo de $R$ é dito um ideal de Lie de $R$ se ele é invariante sob $d_{a}$ para todo $a \in R$.

Proposição 1.6 Seja $D$ anel com divisão e $K$ um subanel com divisão próprio de $D$ tal que $K$ é um ideal de Lie em $D$. Se car $K \neq 2$, então, $K$ está no centro de $D$.

Mais adiante, veremos que os últimos três resultados acima, também valem para comutadores multiplicativos. Veremos, agora, mais uma generalização importante do Teorema de Wedderburn, que é um resultado básico de Herstein para anéis com divisão de característica $p$.

Usaremos a seguinte notação: para um subcorpo $F$ de um anel com divisão $D$, e um subconjunto $S \subseteq D$, escreveremos $F(S)$ para denotar o subanel com divisão de $D$ gerado por $F$ e $S$.

Lema 1.7 (LEMA DE HERSTEIN)- Seja D um anel com divisão de característica $p>0$. Suponhamos que a é um elemento de torção de $D^{*}$ e não central. Então existe $y \in D^{*}$ tal que yay ${ }^{-1}=a^{i} \neq a$ para algum $i>0$. Além disso, y pode ser tomado como sendo um comutador aditivo em $D$.

Teorema 1.8 Seja $D$ um anel com divisão. Se para cada elemento $a \in D$ existir um número natural $n=n(a)>1$ tal que $a^{n}=a$, então, $D$ é comutativo.

$\mathrm{Na}$ verdade, mais tarde foi demonstrado que o resultado acima é verdadeiro para qualquer anel, e é o resultado conhecido por Teorema de Jacobson, cuja prova encontrase em [ 4 ], pág. 73.

Teorema 1.9 (TEOREMA DE JACOBSON) Seja $R$ um anel. Se para cada elemento a em $R$ existir um número natural $n=n(a)>1$ tal que $a^{n}=a$, então, $R$ é comutativo.

Uma generalização do Teorema 1.8 que também foi demonstrada, mais tarde, para um anel qualquer é o próximo teorema. 
Teorema 1.10 Seja $D$ um anel com divisão. Se para cada par de elementos $x, y \in D$ existir um número natural $n=n(x, y)>1$ tal que $(x y-y x)^{n}=x y-y x$, então, $D$ é comutativo.

\section{Demonstração:}

Se $x y-y x \in F$, o centro de $D$, para quaisquer $x, y$, então, $D$ é corpo pela Proposição 1.4. Suponhamos, então que existam $b$ e $b^{\prime}$ tais que $a=b b^{\prime}-b^{\prime} b \notin F$.

Para qualquer $c \in F^{*}$ temos

$$
c a=c\left(b b^{\prime}-b^{\prime} b\right)=c\left(b b^{\prime}\right)-c\left(b^{\prime} b\right)=(c b) b^{\prime}-b^{\prime}(c b)
$$

e também é comutador aditivo não nulo, já que $a$ e $c$ são não nulos e $D$ é anel com divisão.

Assim $a$ e $c a$ têm ordem finita; então, existe um inteiro $k>0$ tal que $1=a^{k}=$ $(c a)^{k}=c^{k} a^{k}$ e concluímos que $c^{k}=1$. Logo, $\operatorname{car} F=\operatorname{car} D=p>0$.

Como $a \notin F$ e é de torção, pelo Lema de Herstein existe um comutador aditivo $y \in D^{*}$ tal que $y a y^{-1}=a^{i} \neq a$.

Por hipótese $y$ é de torção em $D^{*}$ e como $y$ normaliza o grupo cíclico $\left\langle a>\subset D^{*}\right.$, temos que $\langle a\rangle\langle y\rangle$ é subgrupo finito de $D^{*}$ e pela Proposição 1.2 esse subgrupo é comutativo. Logo, $a y=y a$ e obtemos a contradição de que $y a y^{-1}=a$.

Então, não pode existir comutador que não está no centro e, portanto $D$ é corpo.

Definição 1.11 Seja $R$ um anel e $K$ um corpo. Um elemento $a \in R$ é dito algébrico sobre $K$, se existem elementos $\alpha_{0}, \alpha_{1}, \ldots, \alpha_{n} \in K$, não todos nulos, tais que

$$
\alpha_{0} a^{n}+\alpha_{1} a^{n-1}+\cdots+\alpha_{n}=0 .
$$

$O$ anel $R$ é algébrico sobre $K$ se todos os seus elementos forem algébricos sobre $K$.

Outro resultado interessante de Jacobson que é, na verdade, um corolário do Teorema 1.8 é o teorema seguinte.

Teorema 1.12 (TEOREMA DE JACOBSON) Seja D uma álgebra com divisão algébrica sobre um corpo finito $F$. Então $D$ é comutativa (e é, então, uma extensão algébrica de F). 


\section{Demonstração:}

Seja $p=\operatorname{car} F$. Logo $|F|=p^{\alpha}$, para algum inteiro $\alpha$. Para qualquer $d \in D, F(d)$ é uma extensão algébrica finita de $F$. Seja $m=[F(d): F]$. Assim $F(d)$ é um corpo finito e $|F(d)|=p^{\alpha m}=n$, então $d^{n}=d$.

Logo, as hipóteses do Teorema 1.8 estão satisfeitas e $D$ é comutativa.

A seguir, enunciaremos os resultados análogos para comutadores multiplicativos.

Teorema 1.13 Seja D um anel com divisão. Se um elemento c de D comuta com todos comutadores multiplicativos em $D$, então, $c$ está no centro do anel.

Teorema 1.14 Seja D um anel com divisão. Se todos seus comutadores multiplicativos sẫo centrais, então, $D$ é um corpo.

A demonstração é uma conseqüência imediata do teorema anterior.

Definição 1.15 Para um par de anéis com divisão $K \subseteq D$, dizemos que $K$ é normal em $D$ se, para qualquer $x \in D^{*}, x K x^{-1} \subseteq K$ (em outras palavras, se $K^{*}$ é um subgrupo normal de $D^{*}$ ).

O próximo enunciado é um famoso resultado análogo multiplicativo à Proposição 1.6, devido a Cartan, Brauer e Hua e que nos traz uma conseqüência agradável:

Teorema 1.16 (TEOREMA DE CARTAN-BRAUER-HUA) Sejam $D$ e $K$ anéis com divisão com $K \subseteq D$. Suponhamos que $K$ é normal em $D$ e $K \neq D$. Então $K$ está no centro de $D$.

Corolário 1.17 Seja $D$ um anel com divisão e $d \in D$ um elemento não central de $D$. Entẫo, D é gerado como um anel com divisão por todos os conjugados de d.

Enunciaremos, ainda, dois resultados que se referem à estrutura de subgrupos do grupo multiplicativo $D^{*}$ de um anel com divisão $D$ :

Definição 1.18 Para qualquer grupo $G$, chamamos de série central superior de $G$ a série

$$
\{1\} \subseteq G_{1} \subseteq G_{2} \subseteq G_{3} \cdots \subseteq G_{n}
$$

onde $G_{1}=Z(G)$, o centro de $G, \frac{G_{2}}{G_{1}}=Z\left(\frac{G}{G_{1}}\right), \cdots$, etc.

$U m$ grupo $G$ é dito nilpotente se $G_{n}=G$ para algum inteiro n. 
Teorema 1.19 Seja $D$ um anel com divisão e $\{1\} \subseteq G_{1} \subseteq G_{2} \subseteq G_{3} \cdots$ a série central superior do grupo $G=D^{*}$. Então, $G_{1}=G_{2}=G_{3}=\cdots$.

Teorema 1.20 O grupo multiplicativo $D^{*}$ de um anel com divisão $D$ é nilpotente se e somente se $D$ é um corpo.

\section{Demonstração:}

Para demonstrarmos a parte somente se, observemos que se $D^{*}=G$ é nilpotente então, $G_{n}=G$ para algum $n$ inteiro. Pelo Teorema 1.19 temos $G_{1}=G_{2}=G_{3}=\cdots=$ $G_{n}=G$. Logo $G=G_{1}=Z(G)$ e, portanto $G$, e conseqüentemente, $D$, é corpo.

Agora, se $D$ é corpo temos $G_{1}=Z(G)=G$, que implica que $D^{*}$ é nilpotente com $n=1$ o que termina a demonstração do teorema.

Definição 1.21 Seja $G$ um grupo e $S$ um subconjunto de $G$. Para $s \in S$, denotamos por $\operatorname{cl}(s)$ o conjunto de todos os conjugados de $s$, isto é, $\operatorname{cl}(s)=\left\{x^{-1} s x \mid x \in\right.$ $G\}$. Denotamos por $c l(S)=\bigsqcup_{s \in S} c l(s)$. O centralizador de $S$ em $G$ é o conjunto $C_{G}(S)=C(S)=\{x \in G \mid x s=s x \forall s \in S\}$. O normalizador de $S$ em $G$ é $o$ conjunto $N_{G}(S)=N(S)=\{x \in G \mid x S=S x\}$.

Vamos precisar, ainda, de mais alguns teoremas :

Teorema 1.22 Seja $x$ um elemento de um grupo $G$; então, $[G: C(x)]=|c l(x)|$, onde $C(x)=C(\{x\})$.

A demonstração desse teorema se encontra em [14 ], pág. 53.

Teorema 1.23 Seja $K$ subanel com divisão próprio de um anel com divisão $D$, onde $D$ tem ordem infinita. Então, $\left[D^{*}: K^{*}\right]=|D|$.

Teorema 1.24 Se G é um grupo abeliano, finitamente gerado, e $H$ é subgrupo de $G$, então, $H$ é finitamente gerado.

A demonstração desse teorema se encontra em [14 ], pág. 113.

Teorema 1.25 Todo grupo abeliano, finitamente gerado e de torção, é finito.

A demonstração desse teorema se encontra em [ 11 ], pág. 342. 
Definição 1.26 Um módulo $M$ é chamado noetheriano se ele satisfaz à condição de cadeia ascendente, ou seja, não existe nenhuma seqüência infinita ascendente de módulos $M_{1} \varsubsetneqq M_{2} \varsubsetneqq M_{3} \varsubsetneqq \cdots$ em $M$.

Teorema 1.27 ( TEOREMA DE WEDDERBURN) Seja D um anel com divisão com centro $F$, e seja $A$ uma classe de conjugação de $D$ que é algébrico sobre $F$ com polinômio minimal $f(t) \in F[t]$ de grau $n$. Então, existem $a_{1}, \ldots, a_{n} \in A$ tais que $f(t)=\left(t-a_{n}\right) \cdots\left(t-a_{1}\right) \in D[t]$. Também, $f(t)$ é o produto dos mesmos fatores lineares, permutados ciclicamente. $O$ elemento $a_{1} \in A$ pode ser tomado arbitrariamente.

Vamos introduzir a definição e algumas propriedades da função "norma"sobre uma álgebra $A$ de dimensão finita sobre o corpo $F$.

Definição 1.28 Seja $A$ uma álgebra de dimensão finita sobre o corpo $F$ e seja $u \in A$. Seja $\lambda_{u}: A \rightarrow A$ a multiplicação, à esquerda, por $u$, isto é,

$$
\lambda_{u}(x)=u x
$$

É claro que $\lambda_{u}$ é um endomorfismo de A como F-espaço vetorial.

Entâo, definimos a função norma $N$ sobre $A$ por

$$
N(u)=\operatorname{det} \lambda_{u}
$$

É fácil verificar que a norma definida acima tem as seguintes propriedades.

Proposição 1.29 Seja $A$ uma álgebra de dimensão finita sobre o corpo $F$ e seja $K$ um subcorpo de $F \operatorname{com}[F: K]<\infty$. Se $u, v \in F$ e $a \in K$, então:

1) $N(0)=0$ e $N(1)=1$;

2) Se $u \neq 0$ então $N(u) \neq 0$ e $N\left(u^{-1}\right)=N(u)^{-1}$;

3) $N(u v)=N(u) N(v)$ e $N(a u)=a^{n} N(u)$;

4) Se $A$ for um corpo temos $N_{A / K}(u)=N_{F / K}\left(N_{A / F}(u)\right)$.

Definição 1.30 Referire-mo-nos à propriedade 4) acima como Fórmula da Torre para Normas.

Teorema 1.31 ( TEOREMA DE HILBERT Satz 90) Seja E/F uma extensão cíclica, $\eta$ um gerador de $G a l(E / F)$, isto é, $\operatorname{Gal}(E / F)=<\eta>$. Então $N_{E / F}(c)=1$ se, e somente se, existe $u \in E$ talque $c=u(\eta(u))^{-1}$.

A demonstração desse teorema se encontra em [ 5 ], pág. 286. 
Definição 1.32 Uma álgebra A é dita central simples sobre um corpo $F$ se A é uma álgebra simples tendo $F$ como seu centro.

Teorema 1.33 ( TEOREMA DE SKOLEM-NOETHER) Todo automorfismo de uma álgebra central simples e de dimensão finita, é interno.

A demonstração desse teorema se encontra em [4], pág. 100.

Definição 1.34 Dado um par de anéis $S \subseteq R$, dizemos que $R$ é radical sobre $S$ se, para todo $a \in R, a^{n(a)} \in S$ para algum $n(a) \geq 1$.

Teorema 1.35 ( TEOREMA DE KAPLANSKY) Seja D um anel com divisão que é radical sobre seu centro $F$. Então, $D=F$.

Definição 1.36 Um elemento a numa extensão $K$ de um corpo $F$, de característica $p$, é dito separável sobre $\mathbf{F}$ se ele satisfaz um polinômio sobre $F$ que não tem raízes múltiplas e dizemos que é puramente inseparável sobre $\mathbf{F}$ se para algum $k, a^{p^{k}} \in F$.

Uma extensão $K$ de $F$ é dita separável ( puramente inseparável) sobre $F$ se todos os seus elementos são separáveis (puramente inseparáveis) sobre $F$.

Teorema 1.37 ( TEOREMA DE NOETHER-JACOBSON) Seja D um anel com divisão não comutativo algébrico sobre seu centro $F$. Então, existe um elemento em $D \backslash F$ que é separável sobre $F$.

Teorema 1.38 Seja $D$ um anel com divisão com centro $F$ e seja $\left(D^{*}\right)^{\prime}=D^{\prime}$ o subgrupo derivado de $D^{*}$. Se $D^{\prime}$ é radical sobre $F$, então, $D$ não contém elementos puramente inseparáveis sobre $F$.

A demonstração deste teorema está em [ 2 ], pág.184 e 185.

Teorema 1.39 Seja $D$ um anel com divisão com centro $F$. Se $N$ é um subgrupo normal $e$ de torção de $D^{*}$ então $N \subset F$.

A demonstração desse teorema se encontra em [ 14 ], pág. .

Teorema 1.40 ( TEOREMA DE KAPLANSKY) Seja K uma extensão do corpo F onde $K$ é radical sobre $F$, e seja $P$ o corpo primo de $F$. Então, car $P=p>0$ e, ou $K$ é puramente inseparável sobre $F$ ou $K$ é algébrico sobre $P$. (Conseqüentemente, se estas conclusões valem, então, claramente $K$ é radical sobre $F$ ).

Teorema 1.41 ( TEOREMA DE JACOBSON) Todo anel com divisão algébrico sobre um corpo finito, é comutativo. 


\section{Capítulo 2}

\section{O Grupo Multiplicativo de uma Álgebra com Divisão}

Os resultados do capítulo anterior mostram que, no estudo da comutatividade de anéis, é útil considerar os subgrupos do seu grupo multiplicativo. Antes de enunciarmos o nosso resultado principal mostraremos alguns resultados que se referem a esses subgrupos.

Proposição 2.1 Seja $D$ uma álgebra com divisão com centro $F$. Se existe $x \in D \backslash F$, entãa, $|c l(x)|=|D|$ onde $\operatorname{cl}(x)=\left\{d^{-1} x d \mid d \in D^{*}\right\}$ denota o conjunto de todos os conjugados de $x$.

\section{Demonstração:}

Como existe $x \in D \backslash F$, então, pelo Teorema de Wedderburn, $D$ é infinito e o centralizador de $x$ em $D$ denotado pelo conjunto $C(x)=\{d \in D \mid d x=x d\}$ é um subgrupo próprio de $D^{*}$, caso contrário $x \in F$. Logo podemos utilizar o Teorema 1.22 e teremos que $|c l(x)|=\left[D^{*}: C(x)^{*}\right]$. Assim, pelo Teorema 1.23 concluímos que $|c l(x)|=|D|$.

Este resultado implica em nossa próxima proposição.

Proposição 2.2 O grupo multiplicativo de uma álgebra com divisão não enumerável não pode conter nenhum subgrupo normal não central finitamente gerado.

Demonstração: 
Seja $D$ uma álgebra com divisão com centro $F$. Suponhamos que $N$ seja um subgrupo normal não central de $D^{*}$ e seja $a \in N \backslash F$ onde $F$ é o centro de $D$.

Então, $c l(a)=\left\{d^{-1} a d \mid d \in D^{*}\right\} \subset N$. Logo, como $|c l(a)|=|D|$, pela Proposição acima, temos que $|N|=|D|$.

Assim, $N$ também é não enumerável. Logo, $N$ não é finitamente gerado.

No caso geral, ainda não sabemos se o grupo multiplicativo de uma álgebra com divisão pode conter um subgrupo normal não central finitamente gerado.

Fica fácil, agora, vermos que os subgrupos normais finitos do grupo multiplicativo de uma álgebra com divisão estão sempre no centro de $D$, pois, suponhamos que exista um elemento $a \in N \backslash F$ onde $N$ é subgrupo normal finito de $D^{*}$ e $F$ é o centro de $D$. Como $\operatorname{cl}(a) \subset N$ temos que $|\operatorname{cl}(a)|<|N|$. Mas, pela Proposição 2.1 acima, $|\operatorname{cl}(a)|=\left|D^{*}\right|$, o que nos leva à contradição de que $N$ é infinito.

Enunciaremos, agora, o principal teorema desse capítulo.

Teorema 2.3 Seja $D$ uma álgebra com divisão de dimensão finita sobre seu centro $F$, $e$ seja $D^{*}$ o grupo multiplicativo de $D$. Se $D^{*}$ é finitamente gerado, então, $D$ é comutativa.

\section{Demonstração:}

Como $D^{*}$ é finitamente gerada, existem $s$ elementos $d_{1}, \cdots, d_{s}$ em $D^{*}$ tais que

$$
D^{*}=<d_{1}, \cdots, d_{s}>\text {. }
$$

Seja $D^{\prime}=\left(D^{*}\right)^{\prime}$ o subgrupo derivado de $D^{*}$. Assim, $\frac{D^{*}}{D^{\prime}}$ é gerado pelos elementos $\bar{d}_{1}, \ldots, \bar{d}_{s}$ onde $\bar{d}_{k}=d_{k} D^{\prime}$ e, portanto, $\frac{D^{*}}{D^{\prime}}$ também é finitamente gerado.

Além disso, $\frac{D^{*}}{D^{\prime}}$ é abeliano; portanto, seus subgrupos são finitamente gerados pelo Teorema 1.24.

Consideremos o grupo

$$
\frac{F^{*} D^{\prime}}{D^{\prime}}
$$

Como este grupo é subgrupo de $\frac{D^{*}}{D^{\prime}}$, concluímos que $\frac{F^{*} D^{\prime}}{D^{\prime}}$ é finitamente gerado e, portanto,

$$
\frac{F^{*}}{F^{*} \cap D^{\prime}}
$$

também é finitamente gerado já que

$$
\frac{F^{*} D^{\prime}}{D^{\prime}} \cong \frac{F^{*}}{F^{*} \cap D^{\prime}}
$$


pelo 2o. Teorema do Isomorfismo.

Queremos provar que $F^{*} \cap D^{\prime}$ é finito. Para isto, mostraremos que todo $x \in F^{*} \cap D^{\prime}$ é raiz de um polinômio $x^{n}-1$, onde $n$ é o índice de $F$ em $D$.

Como $x \in F^{*} \cap D^{\prime}$ temos $x=c_{1} c_{2} \ldots c_{r}$, onde $c_{i} \in D^{\prime}$. Seja $c_{i}=a_{i} b_{i} a_{i}{ }^{-1} b_{i}{ }^{-1}$ com $a_{i}, b_{i} \in D^{*}$ então, calculando a norma de $c_{i}$ temos

$$
\begin{aligned}
N\left(c_{i}\right) & =N\left(a_{i} b_{i} a_{i}^{-1} b_{i}^{-1}\right) \\
& =N\left(a_{i}\right) N\left(b_{i}\right) N\left(a_{i}^{-1}\right) N\left(b_{i}^{-1}\right) \\
& =N\left(a_{i}\right) N\left(a_{i}^{-1}\right) N\left(b_{i}\right) N\left(b_{i}^{-1}\right) \\
& =N\left(a_{i} a_{i}^{-1}\right) N\left(b_{i} b_{i}^{-1}\right) \\
& =N(1) N(1)=1
\end{aligned}
$$

Como $N\left(c_{i}\right)=1$ para $\forall i$, temos $N(x)=1$.

Fica fácil, agora, vermos que $x^{n}=1$ onde $n=\operatorname{dim}_{F} D$, já que para $d \in D^{*}$ temos $N(d) \neq 0$ e

$$
N(x d)=N(x) N(d)=N(d)
$$

e por outro lado, como $x \in F$ temos

$$
N(x d)=x^{n} N(d)
$$

Logo $x^{n}=1$ como queríamos e portanto $\left|F^{*} \cap D^{\prime}\right|<\infty$.

Agora, sabemos que $\frac{F^{*}}{F^{*} \cap D^{\prime}}$ é finitamente gerado e $F^{*} \cap D^{\prime}$ é finito.

Suponhamos que $x_{1}, \ldots, x_{s} \in F^{*}$ são tais que $\bar{x}_{1}, \ldots, \bar{x}_{s}$ geram $\frac{F^{*}}{F^{*} \cap D^{\prime}}$.

Então, se $x \in F^{*}$ temos

$$
\bar{x}=\bar{x}_{1}^{n_{1}} \ldots \bar{x}_{s}^{n_{s}}
$$

e daí

$$
x_{1}^{n_{1}} \ldots x_{s}^{n_{s}} x^{-1}=y \in F^{*} \cap D^{\prime} .
$$

Logo,

$$
x=y^{-1} x_{1}^{n_{1}} \ldots x_{s}^{n_{s}},
$$

ou seja, temos que $F^{*}$ é gerado por $\left\{x_{1}, \ldots, x_{s}\right\} \cup\left\{F^{*} \cap D^{\prime}\right\}$.

Logo, $F^{*}$ é finitamente gerado.

Finalmente, vamos provar que $F^{*}$ é finito e aí, como $[D: F]<\infty$ temos que $D$ é finito e, portanto, comutativo pelo clássico Teorema de Wedderburn.

Para provarmos que $F^{*}$ é finito, consideremos 2 casos:

1-) Suponhamos que $\operatorname{car} F=0$. Então, $\mathbb{Q}^{*}$ é subgrupo de $F^{*}$ que é um grupo abeliano finitamente gerado. Logo, chegamos ao absurdo de que $\mathbb{Q}^{*}$ é finitamente gerado. 
2-) Se $\operatorname{car} F=p>0$, suponhamos, em primeiro lugar, que $F$ seja algébrico sobre seu corpo primo $\mathbb{Z}_{p}$.

Seja $x \in F$. Existem $a_{0}, a_{1}, \ldots, a_{m} \in \mathbb{Z}_{p}$ com $a_{m} \neq 0$ tais que $x$ é raiz do polinômio

$$
a_{m} X^{m}+a_{m-1} X^{m-1}+\ldots+a_{1} X+a_{0} .
$$

Então,

$$
a_{m} x^{m}+a_{m-1} x^{m-1}+\ldots+a_{1} x+a_{0}=0,
$$

e daí temos que $x^{m}=a_{m}{ }^{-1}\left(-a_{m-1} x^{m-1}-\ldots-a_{1} x-a_{0}\right)$ e então

$$
\begin{aligned}
\left(x^{m}\right)^{p} & =\left(a_{m}^{-1}\right)^{p}\left(-a_{m-1}^{p}\left(x^{m-1}\right)^{p}-\ldots-a_{1}^{p} x^{p}-a_{0}^{p}\right) \\
& =a_{m}{ }^{-1}\left(-a_{m-1}\left(x^{m-1}\right)^{p}-\ldots-a_{1} x^{p}-a_{0}\right) .
\end{aligned}
$$

Assim, $x^{p}$ é raiz de $\left(^{*}\right)$ e, então $\left(x^{p}\right)^{k}$ é também é raiz de $\left(^{*}\right)$ para todo $k$ e como o polinômio $\left(^{*}\right)$ tem no máximo $m$ raízes distintas, tem que existir $k$ tal que $\left(x^{p}\right)^{k-1}=1$. Logo, $F^{*}$ é de torção.

Temos, então, que $F^{*}$ é um grupo abeliano, de torção e finitamente gerado; logo, $F^{*}$ é finito pelo Teorema 1.25.

Suponhamos, agora, então, que exista $x \in F$, que é transcendente sobre $\mathbb{Z}_{p}$. Temos que $\mathbb{Z}_{p}(x)$ é subcorpo de $F$.

Então, para todo número natural $m$, existe um polinômio irredutível $f_{m}(x)$ de grau $m$ em $\mathbb{Z}_{p}[x]$.

Como $F^{*}$ é um grupo abeliano finitamente gerado, vamos considerá-lo como um $\mathbb{Z}$-módulo finitamente gerado. Daí, segue que $F^{*}$ é um $\mathbb{Z}$-módulo noetheriano.

Seja

$$
G_{i}=<f_{1}(x), f_{2}(x), \ldots, f_{i}(x)>
$$

com $i=1,2, \ldots$ uma cadeia de submódulos de $F^{*}$.

Temos que $G_{1} \varsubsetneqq G_{2} \varsubsetneqq G_{3} \varsubsetneqq \ldots$ é uma cadeia ascendente de subgrupos de $F^{*}$ que não é estacionária, o que contradiz a condição noetheriana.

Logo, só podemos ter car $F=p>0$ e $F$ é algébrico sobre seu corpo primo, o que implica que $F^{*}$ é finito. Assim, terminamos a demostração do nosso principal teorema. 


\section{Capítulo 3}

\section{O Grupo Derivado de um Anel com Divisão}

Neste capítulo veremos que, se em um anel com divisão $D$ os elementos do grupo derivado $D^{\prime}$ de $D^{*}$ são algébricos sobre um subcorpo finito de $D$, então, $D$ é comutativo.

Temos que se $D$ é algébrico sobre seu centro $F$, claramente $D^{\prime}$ será algébrico sobre $F$. Por outro lado, quando $D^{\prime}$ é algébrico sobre $F$, não sabemos, é claro, se $D$ será algébrico sobre $F$.

Neste capítulo daremos algumas propriedades de um anel com divisão $D$ onde os elementos de $D^{\prime}$ são algébricos sobre o centro de $D$, e veremos que alguns dos resultados básicos conhecidos para um anel com divisão algébrico sobre sobre seu centro, podem ser generalizados para um anel com divisão $D$ em que $D^{\prime}$ é algébrico sobre $F$.

Teorema 3.1 Seja $D$ um anel com divisão com centro $F$. Então, para cada elemento $a \in D$ algébrico sobre $F$, existe um inteiro positivo $n(a)$, dependendo de a e um elemento $c_{a} \in D^{\prime} \cap F(a)$ tal que $a^{n(a)}=N_{F(a) / F}(a) c_{a}$ com $N_{F(a) / F}\left(c_{a}\right)=1$ onde $N_{F(a) / F}$ é a norma de $F(a)$ em $F$.

\section{Demonstração:}

Seja $f(t)=t^{n}+b_{1} t^{n-1}+\ldots+b_{n}$ o polinômio minimal de $a$ sobre $F$, onde $b_{i} \in F$, $i=1,2, \ldots, n$ e $n=\operatorname{dim}_{F} F(a)$.

Pelo Teorema de Wedderburn ( Teorema 1.27), $f(t)$ se decompõe completamente sobre $F$, isto é, existem $d_{i} \in D^{*}, i=1,2, \ldots, n-1$ tais que

$$
f(t)=(t-a)\left(t-d_{1} a d_{1}^{-1}\right) \ldots\left(t-d_{n-1} a d_{n-1}^{-1}\right) \in D[t] .
$$


Temos que $\left\{1, a, \ldots, a^{n-1}\right\}$ é uma base de $F(a)$ sobre $F$.

Seja $\lambda: F(a) \rightarrow E n d_{F} F(a)$ a multiplicação à esquerda por $a$, isto é,

$$
\begin{array}{lll}
\lambda(a)=\lambda_{a}: & F & (a) \rightarrow F(a) \\
& \lambda_{a} \quad(x)=a x .
\end{array}
$$

A matriz de $\lambda_{a}$ com respeito à base $\left\{1, a, \ldots, a^{n-1}\right\}$ é a matriz companheira do polinômio $f(t)$. Sabe-se que o polinômio minimal de $\lambda_{a}$ é $f(t)$, que também é o polinômio característico. Logo, como $f$ é o produto de fatores lineares distintos, $\lambda_{a}$ é diagonalizável e vale que o $\operatorname{det} \lambda_{a}$ é o produto das raízes de $f$.

Assim, por $\left({ }^{*}\right)$, e lembrando a definição de norma $N_{F(a) / F}(a)=\operatorname{det} \lambda_{a}(\operatorname{Definição~}$ 1.28), temos

$$
N_{F(a) / F}(a)=\operatorname{det} \lambda_{a}=a d_{1} a d_{1}^{-1} d_{2} a d_{2}^{-1} \ldots d_{n-1} a d_{n-1}^{-1} \in F
$$

Seja $c=c_{1} c_{2} \ldots c_{n-1}$ onde $c_{i}$ são comutadores da forma $c_{i}=a^{i-1}\left(d_{i} a d_{i}^{-1} a^{-1}\right) a^{-(i-1)}$. Daí

$$
\begin{aligned}
a^{n} c & =a^{n} c_{1} c_{2} \ldots c_{n-1} \\
& =a^{n} d_{1} a d_{1}^{-1} a^{-1} a^{1} d_{2} a d_{2}^{-1} a^{-1} a^{-1} \cdots a^{n-2} d_{n-1} a d_{n-1}^{-1} a^{-1} a^{-1} a^{-n+2} \\
& =a^{n} d_{1} a d_{1}^{-1} d_{2} a d_{2}^{-1} d_{3} a d_{3}^{-1} \cdots d_{n-1} a d_{n-1}^{-1} a^{-n+1} \\
& =a^{n-1} N_{F(a) / F}(a) a^{-n+1} \\
& =N_{F(a) / F}(a) .
\end{aligned}
$$

Logo, $a^{n} c=N_{F(a) / F}(a) \in F \subset F(a)$.

Fazendo $c_{a}=c^{-1}$ e $n=n(a)$ obtemos

$$
a^{n(a)}=N_{F(a) / F}(a) c_{a},
$$

onde $c_{a} \in D^{\prime}$, pois $c \in D^{\prime}$ e $c_{a} \in F(a)$, já que

$$
c=\underbrace{a^{-n}}_{\in F(a)} \underbrace{N_{F(a) / F}(a)}_{\in F} \in F(a) .
$$

Logo $c_{a} \in D^{\prime} \cap F(a)$, como queríamos.

Falta apenas provar que $N_{F(a) / F}\left(c_{a}\right)=1$. Para isso, tomamos a norma de ambos os lados de $(* *)$ e obtemos:

$$
N_{F(a) / F}\left(a^{n(a)}\right)=N_{F(a) / F}(\underbrace{N_{F(a) / F}(a)}_{=k \in F} c_{a})
$$


De onde vem que

$$
k^{n(a)}=k^{n(a)} N_{F(a) / F}\left(c_{a}\right)
$$

e, portanto, temos

$$
N_{F(a) / F}\left(c_{a}\right)=1 ;
$$

o que termina a demonstração do teorema.

Aqui, $N_{F(a) / F}(a)=k \in F$; então, $N_{F(a) / F}\left(k c_{a}\right)=\operatorname{det}\left[\lambda_{k c_{a}}\right]=\operatorname{det}\left[k \lambda_{c_{a}}\right]=k^{n} \operatorname{det}\left[\lambda_{c_{a}}\right]$.

Como conseqüências deste teorema para extensões finitas de $F$, podemos provar mais alguns teoremas, tais como:

Teorema 3.2 Seja $D$ um anel com divisão com centro $F$, e seja $F \subset K$ uma extensão finita do corpo $F$ em $D$ com $\operatorname{dim}_{F} K=n$. Então para cada elemento $x \in K^{*}$ existe um elemento $c \in D^{\prime} \cap K$ tal que $x^{n}=N_{K / F}(x) c$, com $N_{K / F}(c)=1$. Além disso, se $K$ é um extensão cíclica de $F$, então c pode ser tomado como um comutador em $D^{\prime}$.

Demonstração:

Seja $r$ o grau de $x$ sobre $F$. Pelo teorema acima, existe $c_{x} \in D^{\prime} \cap F(x)$ tal que

$$
x^{r}=N_{F(x) / F}(x) c_{x}
$$

ou seja,

$$
N_{F(x) / F}(x)=x^{r} c_{x}^{-1} \text {. }
$$

Agora, usando a "fórmula da torre para normas"( Definição 1.30) obtemos:

$$
\begin{aligned}
N_{K / F}(x) & =N_{F(x) / F}\left(N_{K / F(x)}(x)\right) \\
& =N_{F(x) / F}\left(x^{\frac{n}{r}}\right) \\
& =\left(N_{F(x) / F}(x)\right)^{\frac{n}{r}} \\
& =\left(x^{r} c_{x}^{-1}\right)^{\frac{n}{r}} \\
& =x^{n} c_{x}^{\frac{-n}{r}}
\end{aligned}
$$

já que $[K: F(x)]=\frac{n}{r}$ e $x^{r}$ e $c_{x}^{-1} \in F(x)$.

Logo,

$$
N_{K / F}(x)=x^{n} c_{x}^{\frac{-n}{r}}
$$

donde temos que

$$
x^{n}=c_{x}{ }^{\frac{n}{r}} N_{K / F}(x) .
$$


Seja $c=c_{x}{ }^{\frac{n}{r}} \in D^{\natural} \cap K$. Pelo Teorema 3.1 temos que $N_{K / F}(c)=1$, o que completa a primeira parte da prova.

Finalmente, seja $G=<\eta>$ o grupo de Galois de $K$ sobre $F$. Como $N_{K / F}(c)=1$, pelo Torema 1.31 de Hilbert podemos encontrar um $u \in K^{*} \subset D^{*}$ tal que

$$
c^{-1}=u(\eta(u))^{-1}
$$

Assim,

$$
c=\eta(u) u^{-1} \text {. }
$$

Agora, o Teorema 1.33 de Skolem-Noether, implica na existência de $v \in K \subset D^{*}$ tal que

$$
\eta(u)=v u v^{-1} \text {. }
$$

Assim, $c=v u v^{-1} u^{-1} \in D^{\prime}$, o que completa a demonstração.

O resultado seguinte é uma generalização do Teorema 1.35 devido a Kaplansky:

Teorema 3.3 Seja $D$ uma álgebra com divisão algébrica sobre seu centro F. Se o grupo derivado $D^{\prime}$ é radical sobre $F$, então $D$ é comutativa.

\section{Demonstração:}

Pelo Teorema 3.1, para cada elemento $x \in D$ existe um inteiro $m(x)$ e um elemento $c_{x} \in D^{\prime} \cap F(x)$ tal que $x^{m(x)}=N_{F(x) / F}(x) c_{x}$. Então,

$$
x^{m(x) n\left(c_{x}\right)}=\left[N_{F(x) / F}(x)\right]^{n(c x)} c_{x}^{n\left(c_{x}\right)} \in F,
$$

ou seja, para cada $x \in D$ existe um inteiro $t(x)=m(x) n\left(c_{x}\right)$ tal que $x^{t(x)} \in F$.

Logo, $D$ é radical sobre $F$, e aplicando o Teorema 1.35 de Kaplansky completamos a demonstração.

Mais tarde (Teorema 3.5), mostraremos que a hipótese de $D$ ser algébrico sobre seu centro é supérflua.

O próximo resultado é uma generalização do Teorema 1.37 de Noether-Jacobson:

Teorema 3.4 Seja D uma álgebra com divisão não-comutativa algébrica sobre seu centro $F$. Então existe um elemento no grupo derivado $D^{\prime}$ que não está em $F$ que é separável sobre $F$. 


\section{Demonstração:}

Se todo elemento em $D^{\prime}$ é puramente inseparável sobre $F$, então, $D^{\prime}$ é radical sobre $F$, o que implica na comutatividade de $D$ pelo Teorema 3.8, que é um absurdo. Logo, existe um elemento de $D^{\prime}$ que não está em $F$ que é separável sobre $F$.

O nosso próximo resultado é uma generalização do Teorema 3.8, como também uma generalização do Teorema 1.35 de Kaplansky, para um anel com divisão $D$ em que $D^{\prime}$ é radical sobre $F$.

Teorema 3.5 Seja $D$ um anel com divisão com centro $F$. Se o grupo derivado $D^{\prime} e ́$ radical sobre $F$, então $D$ é comutativo.

\section{Demonstração:}

Se cada elemento de $D^{\prime}$ é central, então, em particular, cada comutador $x y x^{-1} y^{-1}$ é central. Logo, $D$ é comutativo pelo Teorema 1.14. Então, assumimos que existe um elemento $g \in D^{\prime} \backslash F$ e consideremos $F(g)$, o subcorpo de $D$ gerado por $F$ e $g$.

Como $D^{\prime}$ é radical sobre $F$, existe um inteiro positivo $n(g)$ tal que $g^{n(g)} \in F$. Então, $g$ é algébrico sobre $F$ e seja $m=\operatorname{dim}_{F} F(g)$.

Pelo Teorema 3.2, para cada elemento $x \in F(g)$ existe um elemento $c_{x} \in D^{\prime} \cap F(g)$ tal que $x^{m}=N_{F(g) / F}(x) c_{x}$.

Como $D^{\prime}$ é radical sobre $F$, existe um inteiro positivo $n\left(c_{x}\right)$ tal que $c_{x}^{n\left(c_{x}\right)} \in F$. Então,

$$
x^{m n\left(c_{x}\right)}=\underbrace{\left[N_{F(g) / F}(x)\right]}_{\in F}{ }^{n\left(c_{x}\right)} \underbrace{c_{x}^{n\left(c_{x}\right)}}_{\in F} \in F,
$$

isto é, $F(g)$ é uma extensão radical.

Pelo Teorema 1.40, também de Kaplansky, temos que $\operatorname{car} D=p>0$ e, ou $F(g)$ é puramente inseparável sobre $F$ ou $F(g)$ é algébrico sobre o corpo primo $P$ de $F$.

Mas, $F(g)$ não pode ser puramente inseparável sobre $F$ pelo Teorema 1.38. Suponha, então, que $F(g)$ seja algébrico sobre o corpo primo $P$ de $F$. Então, é claro que $F$ também é algébrico sobre $P$. Como $D^{\prime}$ é algébrico sobre $F$ e $F$ é algébrico sobre $P$, concluímos que $D^{\prime}$ é algébrico sobre $P$.

Assim, se $g \in D^{\prime}$, temos que $P(g)$ é finito (pois, como $P$ é finito e $g$ é algébrico sobre $P$ temos que $\operatorname{dim}_{P} P(g)<\infty$ e daí, $P(g)$ tem que ser finito).

Logo, $P(g)^{*}$ é um grupo multiplicativo finito. Portanto, $g$ tem ordem finita para cada $g \in D^{\prime}$. Isto implica de $D^{\prime}$ é de torção. 
Agora, pelo Teorema 1.39, como $D^{\prime}$ é normal em $D^{*}$ e de torção então $D^{\prime} \subset F$, que é uma contradição para o $g$ escolhido. Logo, todo elemento de $D^{\prime}$ é central, o que implica em $D$ comutativo.

Este teorema nos possibilita deduzir o seguinte resultado que é uma generalização do Teorema 1.37 de Noether-Jacobson, como também uma generalização do Teorema 3.4 para anéis com divisão onde $D^{\prime}$ é algébrico sobre $F$ em vez de $D$ ser algébrico sobre $F$.

Teorema 3.6 Seja $D$ um anel com divisão não comutativo com centro $F$ onde $D^{\prime} e ́$ algébrico sobre $F$. Então existe um elemento no grupo derivado $D^{\prime}$ que não está em $F$ e que é separável sobre $F$.

\section{Demonstração:}

Assumimos, por contradição, que cada elemento de $D^{\prime}$ é puramente inseparável sobre $F$. Então, $D^{\prime}$ é radical sobre $F$. Agora, usando o Teorema 3.5 temos que $D$ é comutativo, o que é uma contradição.

Logo, existe um elemento no grupo derivado $D^{\prime}$ que não está em $F$ que é separável sobre $F$.

O próximo Teorema é uma generalização do Teorema 1.41 de Jacobson e também uma aplicação do Teorema 3.5.

Teorema 3.7 Seja $D$ um anel com divisão onde $D^{\prime}$ é algébrico sobre um subcorpo finito $K$ de $D$. Então, $D$ é comutativo.

\section{Demonstração:}

Seja $\operatorname{car} K=p>0$. Então, para cada $g \in D^{\prime}$ temos que $K(g)$ é um corpo finito, já que $g$ é algébrico sobre $K$ e $K$ é finito. Logo, todo elemento de $K(g)^{*}$ tem ordem finita. Assim, existe um inteiro $m \geq 0$ tal que $g^{m}=1 \in F$ e, portanto, $g$ é radical sobre $F$. Logo, $D^{\prime}$ é radical e até de torção sobre $F$. Usamos, então, o Teorema $3.5 \mathrm{e}$ completamos a demonstração.

Teorema 3.8 Seja $D$ anel com divisão com centro $F$. Se cada elemento de $D^{\prime} e ́$ algébrico sobre $F$, então $D$ é algébrico sobre $F$. Equivalentemente, ou $D$ é algébrico sobre $F$, ou $D^{\prime}$ contém um elemento transcendente sobre $F$. 


\section{Demonstração:}

Sabemos, pelo Teorema de Cartan-Brauer-Hua ( Teorema 1.16 ), que o anel com divisão gerado por $F$ e $D^{\prime}$ é o próprio $D$. Então, é suficiente mostrarmos que a soma e o produto de quaisquer dos dois elementos algébricos de $D$ sobre $F$, são também algébricos sobre $F$.

Vamos provar primeiro que se $x, y \in D$ são algébricos sobre $F$, então $x y$ é algébrico sobre $F$.

Consideremos o grupo $\frac{D^{*}}{F^{*} D^{\prime}}$. Como $x$ e $y$ são algébricos sobre $F$, pelo Teorema 3.1, se $x \in D$ é algébrico sobre $F$, existe um inteiro positivo $n(x)$, dependendo de $x$ e um elemento $c_{x} \in D^{\prime} \cap F(x)$ tal que $x^{n(x)}=N_{F(x) / F}(x) c_{x}$. $F^{*} D^{\prime}$.

Traduzindo isso para o grupo $\frac{D^{*}}{F^{*} D^{\prime}} \operatorname{temos}(x)^{n(x)}=\left(x F^{*} D^{\prime}\right)^{n(x)}=\left(N_{F(x) / F}(x) c_{x}\right)^{n(x)} F^{*} D^{\prime}=$

Logo $x\left(F^{*} D^{\prime}\right)$ é de torção.

Agindo da mesma maneira com um elemento $y \in D$ que é algébrico sobre $F$, concluímos que $y\left(F^{*} D^{\prime}\right)$ também é de torção em $\frac{D^{*}}{F^{*} D^{\prime}}$.

Como $\frac{D^{*}}{F^{*} D^{\prime}}$ é abeliano, temos que $x y\left(F^{*} D^{\prime}\right)$ é de torção, o que implica que $(x y)^{m} \in$ $F^{*} D^{\prime}$. Logo $x y$ é algébrico sobre $F$.

Como a multiplicação de dois elementos algébricos sobre $F$ é também algébrico sobre $F$, concluímos que $x+y=x\left(1+x^{-1} y\right)$ é também algébrico sobre $F$ e terminamos a demonstração do teorema.

O nosso próximo Teorema é também uma aplicação do Teorema 3.1 e diz respeito ao grupo $D^{*}$ e ao seu subgrupo $D^{\prime}$.

Teorema 3.9 Seja $D$ uma álgebra com divisão algébrica sobre seu centro $F$. Se $D^{*} e ́$ finitamente gerado, então $D^{\prime}$ é finitamente gerado.

Demonstração:

Suponhamos que $D^{*}$ é o grupo gerado por elementos $x_{1}, x_{2}, \ldots, x_{m}$. Pelo Teorema 3.1 existem números naturais $n_{i}(1 \leq i \leq m)$, tais que $x_{i}^{n_{i}}=r_{i} c_{i}$, onde $r_{i} \in F$ e $c_{i} \in D^{\prime}$.

Seja $K$, subcorpo finito de $F$ gerado pelos elementos $r_{1}, r_{2}, \ldots, r_{m}$ e consideremos o grupo $\frac{D^{*}}{K D^{\prime}}$.

Temos que $\frac{D^{*}}{K D^{\prime}}$ é um grupo abeliano finitamente gerado e de torção. Assim, temos que $\frac{D^{*}}{K D^{\prime}}$ é finito. 
Então $K D^{\prime}$ é finitamente gerado por [ 13 ], página 298, Lema 8.1.31.

Usando o 2o.Teorema do Isomorfismo para grupos, obtemos que $\frac{D^{\prime}}{K \cap D^{\prime}}$ é finitamente gerado, pois, é isomorfo a $\frac{K D^{\prime}}{K}$, que é finitamente gerado.

Por outro lado, $K \cap D^{\prime}$ é finitamente gerado porque é subgrupo de $K$ que é grupo abeliano finitamente gerado.

Assim, temos que ambos $\frac{D^{\prime}}{K \cap D^{\prime}}$ e $K \cap D^{\prime}$ são finitamente gerados e, portanto, $D^{\prime}$ é finitamente gerado, o que termina a demonstração do teorema. 


\section{Bibliografia}

[1] Akbari, S., Mahdavi-Hezavehi, M., Normal Subgroups of $G L_{n}(D)$ are not Finitely Generated, Proc. Amer. Math. Soc. Vol.128, No. 6 (2000), 1627-1632.

[ 2 ] Herstein, I.N., Multiplicative Commutators in Division Rings, Israel Journal of Math. Vol.31, No. 2, (1978), 180-188.

[ 3 ] Herstein, I.N., Tópicos de Álgebra, S. Paulo, Editora da Universidade e Polígono, 1970.

[4] Herstein, I.N., Noncommutative Rings, Carus Mathematical Monograph 15, New York, Mathematical Association of America, Wiley, 1968. 1974.

[ 5 ] Jacobson, N., Basic Algebra I, São Francisco, W.H. Freeman and Company,

[ 6 ] Kaplansky, Fields and Rings, Chicago, University Press, 1969.

[ 7 ] Lam, T.Y., A First Course in Noncommutative Rings, New York, SpringerVerlag, 1991.

[ 8 ] Mahdavi-Hezavehi, M., Extension of Valuations on Derived Groups of Division Rings, Communications in Algebra, Vol.23(3), (1995), 913-926.

[ 9 ] Mahdavi-Hezavehi, M., Akbari, S., Mehraabaadi, M., Hajie, H., On Derived Groups of Division Rings II, Communications in Algebra, Vol.23(8), (1995), 2881-2887.

[10] Mahdavi-Hezavehi, M., Extending Valuations to Algebraic Division Algebras, Communications in Algebra, Vol.22(11), (1994), 4373-4378.

[11] Milies, F.C.P., Anéis e Módulos, São Paulo, Publicações do IME-USP, 1972.

[ 12 ] Robinson, D.J.S., A Course in the Theory of Groups, New York, SpringerVerlag, 1996.

[ 13 ] Rowen L. H,Ring Theory, Boston, Academic Press,vol2,1988.

14 ] Scott, W. R., Group Theory, Dover Publication, INC, 1987.

[15] Stewart, J., Galois Theory,London, Chapman and Hall Ltd, 1973.

[16] Zallesskii, A. E., Solvable Groups and Crossed Products, Math. Sb. 67 (109), (1965), No.1, 154-160. 